\title{
Prospect of the use of checkpoint inhibitors in hepatocellular cancer treatments
}

\author{
This article was published in the following Dove Press journal: \\ Cancer Management and Research \\ 9 February 2017 \\ Number of times this article has been viewed
}

\author{
Ali Raufi \\ Maria Tria Tirona \\ Division of Hematology/Oncology, \\ Department of Medicine, \\ Joan C. Edwards School of Medicine \\ at Marshall University, Edward \\ Comprehensive Care Center, \\ Huntington, WV, USA
}

Correspondence: Ali Raufi

Division of Hematology/Oncology,

Department of Medicine, Joan C.

Edwards School of Medicine at Marshall

University, Edward Comprehensive Care

Center Huntington, $1400 \mathrm{Hal}$ Greer Blvd,

Huntington, WV 2570I, USA

$\mathrm{Tel}+\mid 304399655$ |

$\mathrm{Fax}+\mid 304399655$

Email raufi@marshall.edu

\begin{abstract}
Hepatocellular cancer (HCC) is a very fatal disease due to limited therapeutic options as well as due to its association with underlying chronic liver disease in the majority of cases. The immune evasion in HCC signifies a major barrier to the delivery of effective immunotherapy. Sorafenib is the only Food and Drug Administration-approved drug available with an overall response rate of $2 \%-3 \%$ and overall survival of 2.8 months. Chemotherapy has not been used routinely because of the relative refractoriness of advanced HCC. The introduction of immune checkpoint inhibitors (cytotoxic T-lymphocyte antigen 4, programmed death 1, and programmed death-ligand 1) has opened a new horizon for cancer immunotherapy. Future direction in immunotherapy for HCC is to rationally combine it with other treatment modalities, including surgery, radiofrequency ablation, and cytotoxic agents, to maximize its therapeutic efficacy.

Keywords: hepatocellular cancer, immune checkpoint inhibitors, cancer immunotherapy
\end{abstract}

\section{Introduction}

Hepatocellular cancer (HCC) is a very lethal disease because of its association with the underlying chronic liver disease in majority of cases. The treatment options are also limited. Surgical resection is the preferred therapy; however, tumor extent and underlying liver dysfunction make most patients ineligible for resection, leaving liver transplantation as the only other curative alternative. The treatment modalities such as radiofrequency ablation (RFA), transarterial chemoembolization, and systemic therapy are considered in patients who are not candidates for curative option. However, indications are limited and may not be applicable in all settings. Sorafenib ${ }^{1}$ is the only Food and Drug Administration (FDA)-approved drug available with an overall response rate of $2 \%-3 \%$ and overall survival (OS) of 2.8 months. Chemotherapy has not been used routinely because of relative refractoriness to chemotherapy of advanced HCC. FDA approval of ipilimumab, a human cytotoxic T-lymphocyte antigen 4 (CTLA4)-blocking antibody, in 2011, and nivolumab, a programmed death 1 (PD-1) inhibitor, in 2014-2015, for patients with metastatic melanoma has opened a new horizon for immunotherapy in cancer. Immunotherapy is now considered a main treatment option for many solid and hematologic malignancies. Recently, immunotherapy including CTLA-4 and PD-1 inhibitor has shown promising antitumor effects in HCC, a tumor that is considered resistant to traditional forms of chemotherapy.

\section{Role of cellular immune evasive mechanisms in HCC}

The cancer immunogram has recently been proposed by Blank et $\mathrm{al}^{2}$ to better understand the interactions between cancer and immune system. The framework of this 
immunogram is built on seven parameters that determine the effectiveness of immune system. These parameters include 1) recognition of tumor foreignness due to mutational load, 2) the immunological status of the patients, 3) the ability of the immune cell to infiltrate into the tumor, 4) the inhibitory state of the tumor microenvironment such as absence of checkpoints, 5) absence of soluble inhibitors (interleukin 6 [IL-6], C-reactive protein), 6) absence of inhibitory tumor metabolism (lactate dehydrogenase, glucose utilization), and 7) the tumor sensitivity to immune effectors, such as major histocompatibility complex expression and interferon- $\gamma$ (IFN- $\gamma$ ) sensitivity. The significance of these parameters may differ greatly among the patients, with some factors being more dominant than others. Because of the multifactorial nature of cancer-immune interactions, combinations of biomarker assays will be valuable to define the current states of the cancer immunogram. This information will help guide treatment choice both during natural cancer-immune interaction and upon immunotherapy. The intrinsic hepatic microenvironment has made it a relatively immune-tolerogenic organ. Existing data describe multiple immune responses that include modifications in the functional ability of immune cells, change in cytokine level, and the expression of immune receptor or ligand. These immune responses promote HCC progression, therefore suggesting that antitumor immunity may be restored with targeted therapies.

Liver sinusoidal endothelial cells, hepatic dendritic cells, and Kupffer cells, by priming hepatic T-cell in the absence of costimulation, serve as tolerogenic antigen-presenting cells (APCs). This results in defective cytotoxicity and immune tolerance. ${ }^{3,4}$ This function is very significant as liver is persistently exposed to antigens absorbed from the gastrointestinal tract.

The inability of the immune system to recognize liver cancer cells is also explained by other proposed mechanisms. These include increase in regulatory T-cell (Tregs), impairment of CD4+ T-cell functions, upregulation of immune checkpoint pathways (CTLA-4, PD-1), suppression of natural killer (NK) cells, and recruitment of immunosuppressive cells, such as monocyte and neutrophils ${ }^{5-11}$ (Figure 1).

The immune hemostasis is maintained by CD4+CD25+ Tregs. Treg has an ability to suppress antitumor immune responses. The preclinical models have shown that the deficiency of Tregs may exacerbate the autoimmunity-related issues. ${ }^{12,13}$ The association of Treg and malignancies has also been demonstrated in several studies. ${ }^{14,15}$ Similar increment of Tregs was also observed in the peripheral circulation and the tumor tissues of HCC patients. ${ }^{5}$ Shen et al ${ }^{16}$ and Kobayashi et $\mathrm{al}^{17}$ further investigated their association with disease progression and outcome. The poor survival was found to be related to the upregulation of Treg that also leads to the impairment of cytotoxic CD8+ T-cell. 7,18,19

Myeloid-derived suppressor cells (MDSCs) symbolize a diverse immunosuppressive cell population that is produced in response to various tumor-derived cytokines. MDSC accumulation in peripheral circulation and within tumors is driven by

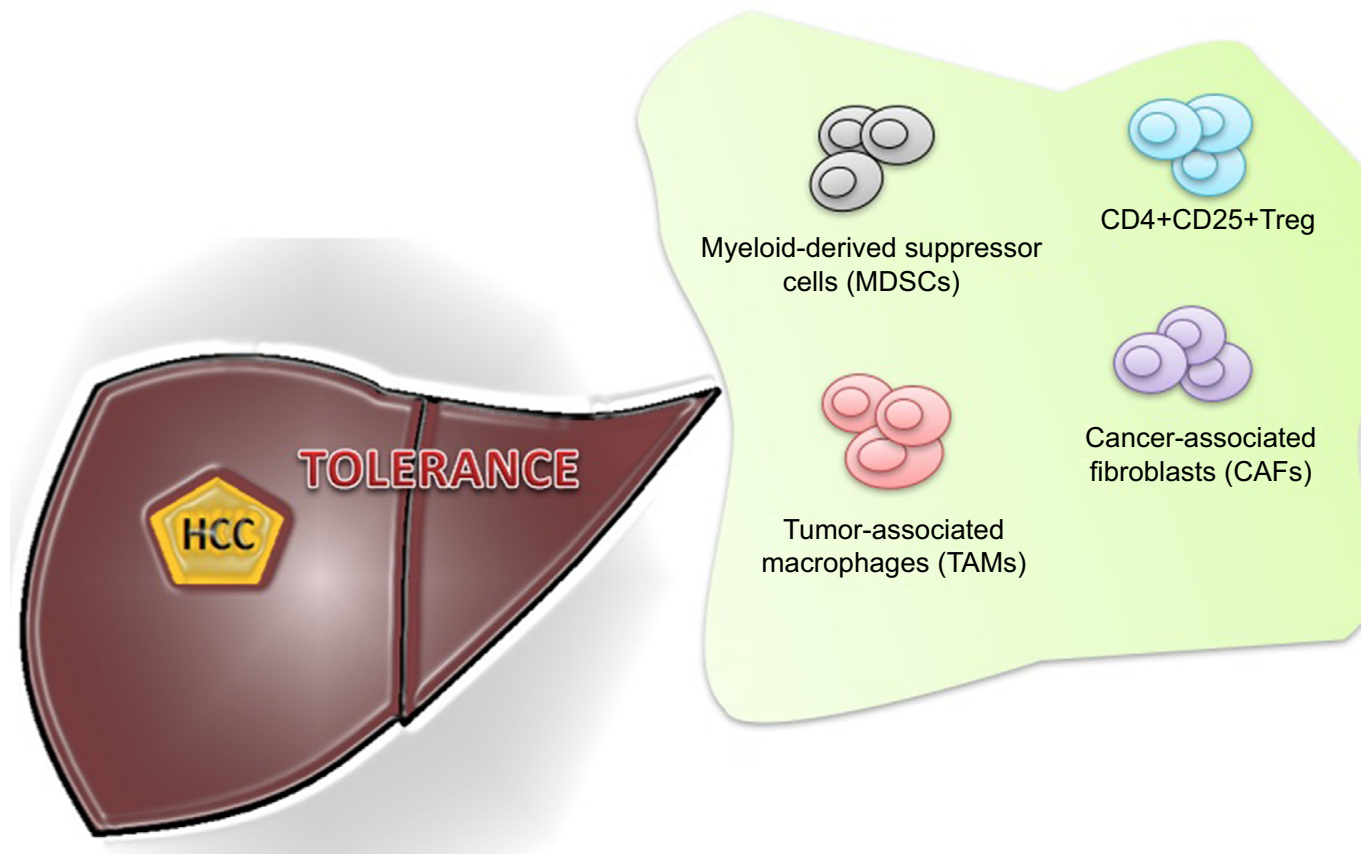

Figure I Immune cells involved in tumor tolerance in hepatocellular cancer (HCC). Abbreviation: Treg, regulatory T-cell. 
increased granulocyte macrophage colony-stimulating factor (GM-CSF) levels produced by cancer cells. ${ }^{20}$ These cells are in different stages of differentiation that constitute myeloid progenitor cells, immature myeloid cells, immature granulocytes, monocytes, macrophages, and dendritic cells. ${ }^{21-23}$ The MDSCs suppress the activity of effector T-cells and impaired NK cell cytotoxicity and cytokine production. ${ }^{8,24}$ Hoechst et al investigated the frequency and function of a CD14+HLA$\mathrm{DR}^{- \text {llow }}$, a new population of MDSC in HCC patients. An MDSC-mediated immune regulatory pathway was also identified in these patients. They proposed that the immunosuppressive function of MDSC is exerted through the induction of CD4+CD25+Foxp3 ${ }^{+}$Tregs in cocultured CD4+ T-cells. ${ }^{24}$

Ilkovitch and Lopez ${ }^{25}$ suggested that liver MDSCs contribute to immunosuppression by interacting with Kupffer cells, resulting in the upregulation of a negative T-cell costimulatory molecule, programmed death-ligand 1 (PD-L1). In HCC patients who received RFA, MDSC has been found to be correlated with tumor progression and prognosis. The recurrence-free survival time was inversely associated with post-therapy frequency of MDSC. ${ }^{26}$

NK cells represent an important cell type whose function is impaired in HCC patients. ${ }^{8}$ It has been suggested that NK cells are inhibited by MDSC through NKp30 receptor. ${ }^{27}$ The induction of systemic immunosuppression by expanding CD14 (+) dendritic cells is another recently identified mechanism in HCC, which may contribute to its progression. These regulatory cells suppress T-cell response by CTLA-dependent IL-10 and indoleamine-2, 3-dioxygenase production in HCC..$^{28}$

The role of tumor-associated macrophages (TAMs) in tumor microenvironment has also been studied. Recent immunological evidence has recognized two distinct states of macrophage activation that have exactly opposite roles in tumor progression and metastasis. M1 macrophages counteract tumorigenesis by enhancing Th1 immunocyte responses and activating other tumor-killing mechanisms. On the other hand, M2 macrophages are immunoregulators, participate in Th2 responses, and enhance tumor growth. TAMs have recently been found to play a significant role in HCC progression and metastasis. ${ }^{29}$ It has been reported that TAMs decrease antitumor immunity by suppressing T-cell function directly by the expression of PD-L1 and therefore contributed to the growth of HCC. Tumor-activated monocytes also secreted cytokines that trigger the proliferation of pro-inflammatory IL-17-producing CD8 (+) T-cells. These IL-17(+) cells are accumulated in $\mathrm{HCC}$ and promote angiogenesis that results in disease progression..$^{30,31}$
The high concentration of TAMs in surgically resected HCC also has prognostic value. TAM was found to be a predictor of poor outcome in most of the studies. ${ }^{19,32}$ The peritumoral infiltration of neutrophils also has been shown to enhance angiogenesis progression. ${ }^{33}$ There is also an increasing evidence that cancer-associated fibroblasts (CAFs) also promote tumorigenesis and metastasis by stimulating angiogenesis, malignant cell survival, and proliferation. CAFs are found in HCC with preexisting chronic hepatitis and liver cirrhosis. These CAFs may be derived from the activation of static fibroblast because of repeated inflammatory stimulation and tissue fibrosis. It is suggested that during course of $\mathrm{HCC}$ progression, fibroblasts in peritumoral tissue progressively convert into CAFs. This progression is accelerated by tumor-secreted lysophostatidic acid, which stimulates the recruitment of peritumor fibroblasts and their differentiation into myofibroblasts. ${ }^{34,35}$

\section{Immune checkpoint inhibitors}

Cancer immunotherapy has been revolutionized by the discovery of two checkpoints, CTLA-4, and the programmed cell death protein 1 pathway (PD-1/PD-L1; Figure 2). Immunotherapy against these negative immunologic regulators has given new paradigm shift to immunotherapy and is now considered a major component of cancer therapeutic modalities. CTLA-4 was the first negative checkpoint regulator to be clinically targeted. Ipilimumab (an anti-CTLA-4 antibody) showed a significant OS in patients with advanced melanoma in randomized Phase III trial, ${ }^{36}$ which led to its approval by the US FDA in 2011.

$\mathrm{HCC}$-induced immune tolerance is associated with multiple immunosuppressive mechanisms. HCC immune tolerance is mediated through decreased costimulatory or increased inhibitory checkpoint signaling that leads into immunosuppression. Significant reductions in expression of costimulatory molecules, such as B7-1 and B7-2, have been identified in $\mathrm{HCC}$ cells, ${ }^{37}$ resulting in a decrease of B7/CD28mediated activation of effector T-cells. Immune-inhibitory receptors and ligands also contribute a crucial role in the maintenance of HCC immune tolerance. The CTLA- $4,{ }^{28}$ PD-1 and its ligand (PD-L1), ${ }^{38}$ lymphocyte-activation gene 3 (LAG-3), ${ }^{39}$ Tim-3 (T-cell membrane protein 3) and its ligand (galectin-9), ${ }^{40}$ and adenosine A2a receptor (A2aR) ${ }^{41}$ are among the several inhibitory checkpoints that have been related with defective immune process in HCC.

LAG-3 is a molecule that has an inhibitory effect on T-cell responses. Li et $\mathrm{al}^{39}$ demonstrated the correlation between LAG-3 expression and HBV-specific CD8+ T-cell 

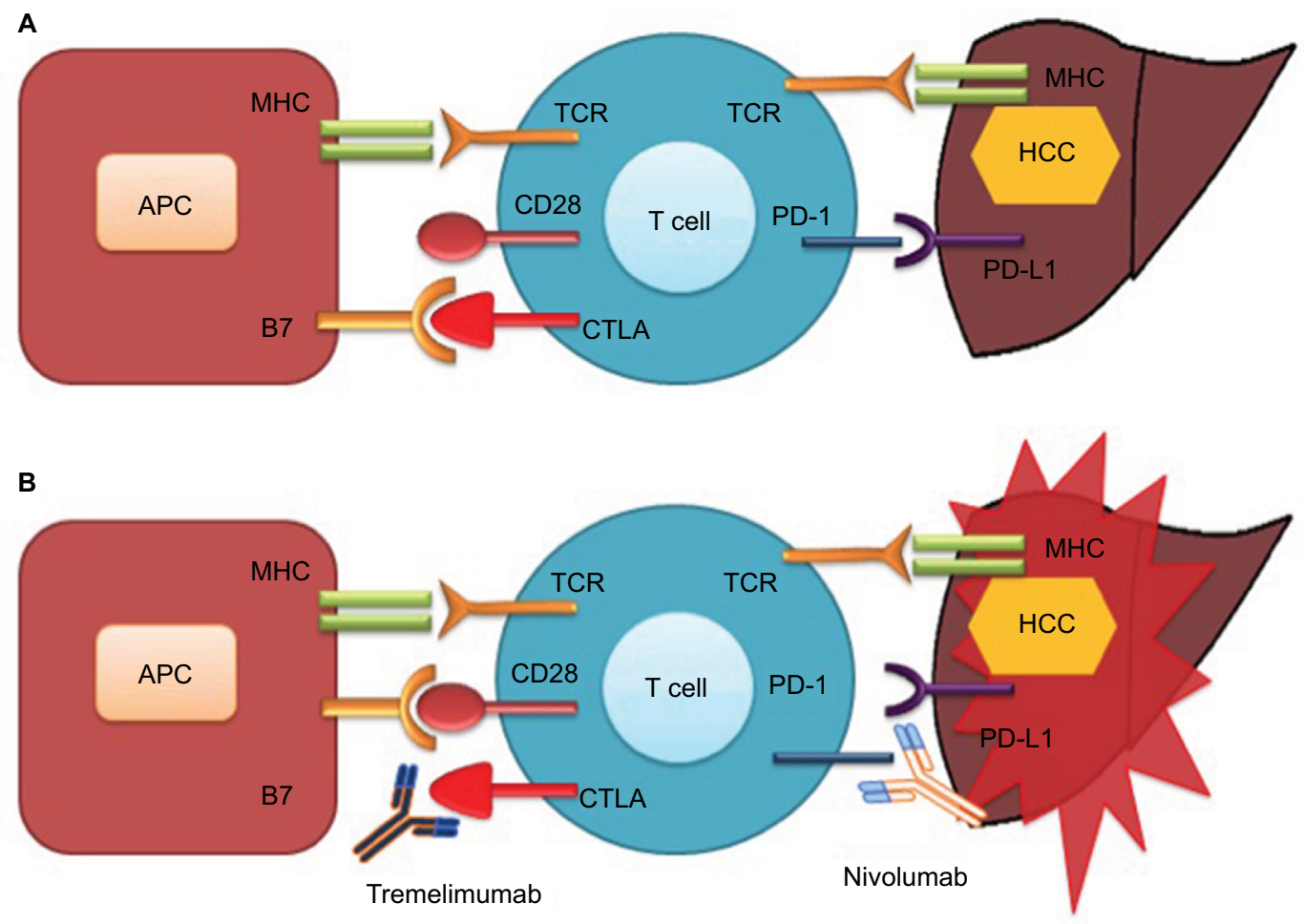

Figure 2 Check point inhibition and hepatocellular cancer

Notes: (A) Interaction of CTLA-4 with B7 and binding of PD-I with PD-LI inhibit T-cell function resulting in tumor proliferation. (B) Antibody-mediated blockade of CTLA4 (tremelimumab) and PD-I (nivolumab) enhanced T-cell function resulting in tumor death.

Abbreviations: APC, antigen-presenting cell; CTLA, cytotoxic T-cell lymphocyte antigen; HCC, hepatocellular cancer; MHC, major histocompatibility complex; PD-I, programmed cell death-I; PD-LI, programmed cell death-ligand I; TCR, T-cell receptor.

malfunction. The upregulation of LAG-3 was found to cause severe impairment in tumor-infiltrating CD8+ T-cells of HCC patients. Li et al ${ }^{40}$ suggested the Tim-3/galectin- 9 signaling pathway as a potential immunotherapeutic target in patients with HBV-associated HCC. Their work revealed that this pathway mediates T-cell dysfunction, which leads to poor prognosis in HCC patients. The glucocorticoid-induced tumor necrosis factor receptor (GITR) and the inducible T-cell costimulator (ICOS) are regulators (stimulatory checkpoint molecules) of the immunosuppressive function of Tregs. Pedroza-Gonzalez et $\mathrm{al}^{42}$ showed that GITR and ICOS are upregulated in Tregs infiltrating HCC, and, therefore, suggested their role as a novel target for immunotherapeutic interventions against tumor progression. In another study, ${ }^{43}$ they suggested that a combination of both GITR engagement and CTLA-4 blockade can improve the antitumor immunity by abrogating tumor-infiltrating Treg-mediated suppression in $\mathrm{HCC}$.

HCC-induced immune tolerance along with tolerogenic hepatic environment and chronic inflammatory conditions justify the use of these immune-targeting agents and checkpoint inhibitors alone or in combination with other treatment modalities in HCC. They are now regarded as promising strategies in clinical development. Table 1 summarizes the immune check inhibitors (CTLA-4, PD-1, and PD-L1) that have been developed and being tested in clinical trials for HCC. The clinical parameters of eligible patients used in clinical studies for anti-CTLA-4 or anti-PD-1/PD-L1 treatment are mentioned in Table 2. This review is focused on the role of these checkpoints and their antibodies relevant for the treatment of patients with HCC.

\section{Role of CTLA-4 in HCC and its clinical implication and progress}

The immune system plays an important role in identifying and eradicating classical highly immunogenic tumors, such as melanoma. The tumor-associated antigens (TAAs) expressed on transformed tumor cells activate the immune system of the body. However, this alone is not sufficient for the initiation of an effective response. The interaction between antitumor T-cells and APCs is central to the development of effective antitumor T-cell immunity. T-cell activation has a complex mechanism that is modulated by stimulatory (cellsurface molecules CD28) and inhibitory CTLA-4 signals. Their collaboration is critical for the coordination of effective immune system's response to a tumor. ${ }^{44-46}$ Moreover, 
Table I Clinical trials on antibodies targeting PD-I or PD-LI in hepatocellular cancer

\begin{tabular}{|c|c|c|c|c|c|}
\hline Antibodies & Class & Phase & Monotherapy/combination & Trial ID & Status \\
\hline \multicolumn{6}{|c|}{ Anti-cytotoxic T-lymphocyte antigen 4} \\
\hline Tremelimumab & Human $\lg G 2$ & II & Monotherapy & NCT0I008358 & Completed \\
\hline Tremelimumab & Human $\lg G 2$ & 1 & Chemoembolization and radiofrequency ablation & NCT0I8536I8 & Recruiting \\
\hline \multicolumn{6}{|l|}{ Anti-PD-I } \\
\hline Nivolumab & Human IgG4 & I & Monotherapy & NCT0I658878 & Recruiting \\
\hline Nivolumab & Human $\lg \mathrm{G} 4$ & III & Monotherapy vs sorafenib & NCT02576509 & Recruiting \\
\hline Nivolumab & Human $\lg G 4$ & 1 & Galunisertib & NCT02423343 & Recruiting \\
\hline \multicolumn{6}{|l|}{ Anti-PD-LI } \\
\hline MEDI4736 & Humanized lgG & 1 & Ramucirumab & NCT02572687 & Recruiting \\
\hline MEDI4736 & Humanized lgG & $\mathrm{I} / \mathrm{II}$ & Monotherapy and with Tremelimumab & NCT02519348 & Recruiting \\
\hline
\end{tabular}

Abbreviations: PD-I, programmed death I; PD-LI, programmed death-ligand I; Ig, immunoglobulin.

Table 2 Clinical parameters of eligible HCC patients for anticytotoxic T-lymphocyte antigen 4 or anti-programmed death I/ programmed death-ligand I treatment

Subjects of 18 years or older (men and women)

Histologically confirmed advanced HCC

They are not eligible for surgical and/or locoregional therapies

They have progressive disease after surgical and/or locoregional therapies

Eastern Cooperative Oncology Group performance status of 0-1 Dose escalation phase: Child-Pugh score of 7 points or less. Cohort 5 : Child-Pugh class B (B7-B8). For all other cohorts Child-Pugh score of 6 points or less

There is no history of autoimmune disease, any prior or current clinically significant ascites, or any history of hepatic encephalopathy

Abbreviation: HCC, hepatocellular cancer.

CD28 facilitates and maintains a T-cell response. TAAs presented in context with APCs bind with T-cell receptors (TCRs). The B7 molecules on the APC surface bind with $\mathrm{CD} 28$ receptors on the T-cell surface and their costimulatory signal leads to the translation of TCR stimulation into T-cell activation. This ultimate activation of T-cells induces T-cell proliferation, cytokine secretion, and gene expression. These T-cell-mediated antitumor immune responses are regulated by inhibitory signals of CLTA- 4 whose expression is upregulated upon TCR stimulation. CTLA-4 prevents the costimulatory signal by competing with $\mathrm{CD} 28$ for binding to $\mathrm{B} 7$ ligands (CD80 and CD86) on the surface of APC because of higher affinity and avidity. ${ }^{47}$ This binding inhibits the T-cell activation and proliferation through a homeostatic mechanism to prevent autoimmunity under physiological conditions ${ }^{48}$ The CTLA-4-deficient mice die from lethal lymphoproliferation as demonstrated in preclinical model. ${ }^{49}$

Unlike melanoma, magnitude of tumor-antigen-specific T-cells in HCC patients is very limited. ${ }^{50,51}$ Flecken et $\mathrm{al}^{50}$ demonstrated the immunodominance and functional alterations of TAA-specific CD8+ T-cell responses in HCC. The IFN- $\gamma$-producing CD8+ T-cells specific for TAA were detected in the periphery as well as in liver and tumor tissue.
Although these cells displayed clear immunodominance pattern, no steady hierarchy was observed between different TAAs. The efficacy of such responses was functionally limited as these TAA-specific CD8+ T-cells were found to be impaired in their ability to produce IFN- $\gamma$.

Chen et $\mathrm{al}^{52}$ studied the role of CTLA- 4 in a subcutaneous murine hepatoma model. They demonstrated the enhancement of antitumor immunity by coadministration of CTLA-4 blockade, intratumoral GM-CSF microspheres, and thermal ablation. Promising results of this observation were further supported upon tumor rechallenge with this regimen. This causes rejection of tumor rechallenge in $90 \%$ of treated mice while cure of established distant tumor was observed in $50 \%$ of the treated mice.

Sangro et $a l^{53}$ evaluated the efficacy and safety of antiCTLA-4 antibodies (tremelimumab) for the treatment of HCC. Eligible patients include HCC patients with liver cirrhosis (classified as Child-Pugh grades A and B) and hepatitis $\mathrm{C}$ who were not the candidates of liver-directed local therapy or had failed the standard of care for HCC. The partial response was observed in $17.6 \%$ of patients (three out of 17 assessable patients) per Response Evaluation Criteria In Solid Tumors (RECIST) criteria, whereas disease control rate was $76.4 \%$. The recorded toxicity profile was tolerable in the study. The patients most frequently developed skin rash (65\%), fatigue (55\%), and anorexia (50\%). A transient elevation of serum transaminase activity was observed only during the initial course of tremelimumab and was not seen with subsequent cycles. Severe immune-mediated adverse events were not recorded and, therefore, none of the patients required the initiation of steroid. The study included the most relevant group of patients (HCC with hepatitis $\mathrm{C}$ virus [HCV]-related cirrhosis); however, results may show differently in chronic HBV patients as far as safety profile is concerned with relatively higher hepatic failure rates than HCV because of frequent inflammatory flares in HBV 
patients, which may eventually result in variable tremelimumab-induced response. ${ }^{54}$ Nevertheless, the demonstration of acceptable toxicity profile of tremelimumab in this study provides the first evidence as one of the potentially new therapeutic approaches of CTLA-4-targeted immunotherapy in $\mathrm{HCC}$ patients.

The role of other alternative immunomodulatory molecules (Tim-3, PD-1, or LAG-3) has been studied to overcome T-cell exhaustion during chronic viral infection. ${ }^{55,56}$

\section{Role of PD-I/PD-LI in HCC and its clinical implication and progress}

The promising results in targeting CTLA-4 in cancers such as melanoma have attracted researchers to approach and target other immunologic checkpoints including PD-1/PD-L1.

PD-1 is an immune-inhibitory receptor that modulates the activity of T-cells at different stages of the immune response through interaction with its ligands, PD-L1 (B7-H1) and PD-L2 (B7-DC). ${ }^{57-59}$

PD-1/PD-L1 is expressed on T-cells, activated B cells, monocytes, Tregs, and NK cells. The PD-1/PD-L1 interaction inhibits T-lymphocyte proliferation and functions (cytotoxicity, cytokine release), inhibits apoptosis of the tumor cells, induces apoptosis of tumor-specific T-cells and promotes the conversion of effector cells into Treg cells. ${ }^{60-62}$

Unlike CTLA-4 that modulates the immune responses of early activation of naive and memory T-cells following TCR engagement, PD-1 mostly inhibits effector T-cell activity in peripheral tissues in response to infection or tumor progression. ${ }^{63}$

Limited data are available with respect to the preclinical evaluation in HCC. Zabala et $\mathrm{al}^{10}$ evaluated the antitumor effect of IL-12-based gene therapy on HCC occurring spontaneously in mice. A plasmid vector expressing IL-12 in a liverspecific and doxycycline-inducible manner was transferred to the liver of L-PK/c-myc mice with HCC. Doxycycline administration increased serum IL-12 and IFN- $\gamma$ and induced tumor lymphocytic infiltration resulting in tumor stabilization or regression in less than half of all treated mice. The tumor resistance in nonresponder mice was found to be correlated to increase in Tregs and higher expression of the immuneinhibitory molecules, such as PD-1 and PD-L1. Willimsky et $\mathrm{al}^{64}$ established a transgenic murine model of virus-induced HCC by hepatocyte-specific adenovirus-induced activation of the oncogenic SV40 large T antigen. They demonstrated progressive local immune inhibition with considerably greater T-cell infiltration in early-stage HCC compared with that in advanced-stage HCC.
PD-1/PD-L1 contributed to local tumor-antigen-specific tolerance and was expressed in all CD8+ T-cells and HCC.

The expression of PD-1 by T-cells has prognostic relevance. Persistent expression is highly suggestive of an exhausted phenotype resulting from a decrease in effector function. ${ }^{65,66}$ Shi et $\mathrm{l}^{38}$ described circulating and intratumor PD-1/PD-L1 expression and analyzed their association with disease progression in HBV-infected patients. They found out that the frequency of circulating PD-1+/CD8+ T-cells increased with disease progression. Moreover, PD-1 expression was found to be significantly increased in tumorinfiltrating effector CD8+ T-cells. The higher expression of PD-1 was correlated with significantly poorer prognosis. Gao et $\mathrm{al}^{67}$ investigated the expression of PD-L1 in human HCC to define its prognostic significance after curative surgery. Results showed that patients with higher expression of PD-L1 had a significantly poorer prognosis than patients with lower expression. Multivariate analysis revealed tumor expression of PD-L1 as an independent predictor for postoperative recurrence. In addition, tumor-infiltrating cytotoxic and Tregs were also found to be independent prognostic factors for both survival and recurrence.

Recently, El-Khoueiry et al ${ }^{68}$ presented Phase I/II data of patients with advanced HCC in American Society of Clinical Oncology (ASCO) 2015 annual meeting. The authors demonstrated safety and antitumor activity of nivolumab, a fully human IgG4 monoclonal antibody PD-1 inhibitor in advanced HCC. Nivolumab was administered intravenously every 2 weeks for up to 2 years. The dose range was from 0.1 to $10 \mathrm{mg} / \mathrm{kg}$. Although there was a relatively small sample study size of 42 patients, the results compare favorably with the existing treatment options. Eleven patients had HCV and 10 patients had HBV infection, while $50 \%$ of patients were unaffected. Approximately $19 \%$ of patients (eight out of 42 eligible patients) responded to nivolumab. Among the responders, two patients achieved complete response, while six patients had partial response. The responses to nivolumab have been durable in $75 \%$ responding patients (six out of eight) and were observed across all dose levels and etiologic cohorts. Overall, approximately half of the total evaluable patients ( 20 out of 42 ) had stable disease with the longest-lasting 17 months. In addition, at 12 months, $62 \%$ of patients in the study were still surviving with HCC. No maximum-tolerated dose was found. Treatment-related adverse events of any grade occurred in $68 \%$ patients. Grade $3 / 4$ adverse events were observed in $19 \%$ patients. The most common ( $\geq 5 \%$ patients) grade 3 adverse events were increases in aspartate aminotransferase (11\%) and alanine 
aminotransferase $(9 \%)$, while treatment-related grade 4 events consisted of elevated lipase (2\%).

Sorafenib, a tyrosine kinase inhibitor, is currently the only FDA-approved drug for advanced HCC. In comparison to this agent in treating $\mathrm{HCC}$, nivolumab showed promising and encouraging OS results even in $70 \%$ patients who received prior sorafenib therapy. The response rate was $19 \%$ with nivolumab as compared to $2 \%-3 \%$ with sorafenib. There was also $20 \%$ OS difference at 12 months $(62 \%$ in patient with nivolumab vs $42 \%$ with sorafenib). The patients with $\mathrm{HCC}$, including those with HBV and HCV infection, had manageable safety profile with nivolumab, and this safety profile was similar to other tumor types treated with nivolumab. However, these findings of nivolumab in HCC need to be validated in a large randomized Phase III trial.

\section{Conclusion}

$\mathrm{HCC}$ is a lethal cancer with limited therapeutic options. The immune evasion in HCC signifies a major barrier to the delivery of effective immunotherapy. The introduction of checkpoint regulators (CTLA-4, PD-1, and PD-L1) and their blockade has opened a new horizon for cancer immunotherapy. Emerging data in HCC suggest that circumventing these immunosuppressive pathways can lead to promising objective clinical responses with acceptable toxicity profile. Future direction in immunotherapy for $\mathrm{HCC}$ is to rationally combine it with other treatment modalities, including surgery, RFA, and cytotoxic agents, to maximize its therapeutic efficacy.

\section{Disclosure}

The authors report no conflicts of interest in this work.

\section{References}

1. Llovet JM, Ricci S, Mazzaferro V, et al. Sorafenib in advanced hepatocellular carcinoma. N Engl J Med. 2008;359(4):378-390.

2. Blank CU, Haanen JB, Ribas A, Schumacher TN. Cancer immunology. The "cancer immunogram". Science. 2016;352(6286):658-660.

3. Bowen DG, Zen M, Holz L, Davis T, McCaughan GW, Bertolino $\mathrm{P}$. The site of primary $\mathrm{T}$ cell activation is a determinant of the balance between intrahepatic tolerance and immunity. $J$ Clin Invest 2004;114(5):701-712.

4. Thomson AW, Knolle PA. Antigen-presenting cell function in the tolerogenic liver environment. Nat Rev Immunol. 2010;10(11):753-766.

5. Ormandy LA, Hillemann T, Wedemeyer H, Manns MP, Greten TF, Korangy F. Increased populations of regulatory $\mathrm{T}$ cells in peripheral blood of patients with hepatocellular carcinoma. Cancer Res. 2005;65(6):2457-2464.

6. Chen KJ, Lin SZ, Zhou L, et al. Selective recruitment of regulatory $\mathrm{T}$ cell through CCR6-CCL20 in hepatocellular carcinoma fosters tumor progression and predicts poor prognosis. PLoS One. 2011;6(9):e24671.

7. Gao Q, Qiu SJ, Fan J, et al. Intratumoral balance of regulatory and cytotoxic $\mathrm{T}$ cells is associated with prognosis of hepatocellular carcinoma after resection. J Clin Oncol. 2007;25(18):2586-2593.
8. Hoechst B, Voigtlaender T, Ormandy L, et al. Myeloid derived suppressor cells inhibit natural killer cells in patients with hepatocellular carcinoma via the NKp30 receptor. Hepatology. 2009;50(3):799-807.

9. Alisa A, Ives A, Pathan AA, et al. Analysis of CD4+ T-cell responses to a novel alpha-fetoprotein-derived epitope in hepatocellular carcinoma patients. Clin Cancer Res. 2005;11(18):6686-6694.

10. Zabala M, Lasarte JJ, Perret C, et al. Induction of immunosuppressive molecules and regulatory $\mathrm{T}$ cells counteracts the antitumor effect of interleukin-12-based gene therapy in a transgenic mouse model of liver cancer. J Hepatol. 2007;47(6):807-815.

11. Greten TF, Ormandy LA, Fikuart A, et al. Low-dose cyclophosphamide treatment impairs regulatory $\mathrm{T}$ cells and unmasks AFP-specific CD4+ T-cell responses in patients with advanced HCC. J Immunother. 2010;33(2):211-218.

12. Wildin RS, Ramsdell F, Peake J, et al. X-linked neonatal diabetes mellitus, enteropathy and endocrinopathy syndrome is the human equivalent of mouse scurfy. Nat Genet. 2001;27(1):18-20.

13. Bennett CL, Christie J, Ramsdell F, et al. The immune dysregulation, polyendocrinopathy, enteropathy, $\mathrm{X}$-linked syndrome (IPEX) is caused by mutations of FOXP3. Nat Genet. 2001;27(1):20-21.

14. Unitt E, Rushbrook SM, Marshall A, et al. Compromised lymphocytes infiltrate hepatocellular carcinoma: the role of T-regulatory cells. Hepatology. 2005;41(4):722-730.

15. Yang XH, Yamagiwa S, Ichida T, et al. Increase of CD4+CD25+ regulatory T-cells in the liver of patients with hepatocellular carcinoma. J Hepatol. 2006;45(2):254-262.

16. Shen X, Li N, Li H, Zhang T, Wang F, Li Q. Increased prevalence of regulatory $\mathrm{T}$ cells in the tumor microenvironment and its correlation with TNM stage of hepatocellular carcinoma. J Cancer Res Clin Oncol. 2010;136(11):1745-1754.

17. Kobayashi N, Hiraoka N, Yamagami W, et al. FOXP3+ regulatory T cells affect the development and progression of hepatocarcinogenesis. Clin Cancer Res. 2007;13(3):902-911.

18. Fu J, Xu D, Liu Z, et al. Increased regulatory $\mathrm{T}$ cells correlate with CD8 T-cell impairment and poor survival in hepatocellular carcinoma patients. Gastroenterology. 2007;132(7):2328-2339.

19. Zhou J, Ding T, Pan W, Zhu LY, Li L, Zheng L. Increased intratumoral regulatory $\mathrm{T}$ cells are related to intratumoral macrophages and poor prognosis in hepatocellular carcinoma patients. Int $J$ Cancer. 2009;125(7):1640-1648.

20. Mueller MM, Fusenig NE. Constitutive expression of G-CSF and GMCSF in human skin carcinoma cells with functional consequence for tumor progression. Int J Cancer. 1999;83(6):780-789.

21. von Boehmer H. Mechanisms of suppression by suppressor T cells. Nat Immunol. 2005;6(4):338-344.

22. Greten TF, Manns MP, Korangy F. Myeloid derived suppressor cells in human diseases. Int Immunopharmacol. 2011;11(7):802-807.

23. Gabrilovich DI, Ostrand-Rosenberg S, Bronte V. Coordinated regulation of myeloid cells by tumours. Nat Rev Immunol. 2012;12(4):253-268.

24. Hoechst B, Ormandy LA, Ballmaier M, et al. A new population of myeloid-derived suppressor cells in hepatocellular carcinoma patients induces CD4(+)CD25(+)Foxp3(+) T cells. Gastroenterology. 2008;135(1):234-243.

25. Ilkovitch D, Lopez DM. The liver is a site for tumor-induced myeloidderived suppressor cell accumulation and immunosuppression. Cancer Res. 2009;69(13):5514-5521.

26. Arihara F, Mizukoshi E, Kitahara M, et al. Increase in CD14+HLA-DR -/low myeloid-derived suppressor cells in hepatocellular carcinoma patients and its impact on prognosis. Cancer Immunol Immunother. 2013;62(8):1421-1430.

27. Cai L, Zhang Z, Zhou L, et al. Functional impairment in circulating and intrahepatic NK cells and relative mechanism in hepatocellular carcinoma patients. Clin Immunol. 2008;129(3):428-437.

28. Han Y, Chen Z, Yang Y, et al. Human CD14+ CTLA-4+ regulatory dendritic cells suppress T-cell response by cytotoxic T-lymphocyte antigen-4-dependent IL-10 and indoleamine-2,3-dioxygenase production in hepatocellular carcinoma. Hepatology. 2014;59(2):567-579. 
29. Shirabe K, Mano Y, Muto J, et al. Role of tumor-associated macrophages in the progression of hepatocellular carcinoma. Surg Today. 2012;42(1):1-7.

30. Kuang DM, Zhao Q, Peng C, et al. Activated monocytes in peritumoral stroma of hepatocellular carcinoma foster immune privilege and disease progression through PD-L1. J Exp Med. 2009;206(6):1327-1337.

31. Kuang DM, Peng C, Zhao Q, et al. Tumor-activated monocytes promote expansion of IL-17-producing CD8+ T cells in hepatocellular carcinoma patients. J Immunol. 2010;185(3):1544-1549.

32. Zhu XD, Zhang JB, Zhuang PY, et al. High expression of macrophage colony-stimulating factor in peritumoral liver tissue is associated with poor survival after curative resection of hepatocellular carcinoma. J Clin Oncol. 2008;26(16):2707-2716.

33. Kuang DM, Zhao Q, Wu Y, et al. Peritumoral neutrophils link inflammatory response to disease progression by fostering angiogenesis in hepatocellular carcinoma. J Hepatol. 2011;54(5):948-955.

34. Mazzocca A, Dituri F, Lupo L, Quaranta M, Antonaci S, Giannelli G. Tumor-secreted lysophostatidic acid accelerates hepatocellular carcinoma progression by promoting differentiation of peritumoral fibroblasts in myofibroblasts. Hepatology. 2011;54(3):920-930.

35. Kojima Y, Acar A, Eaton EN, et al. Autocrine TGF-beta and stromal cell-derived factor-1 (SDF-1) signaling drives the evolution of tumorpromoting mammary stromal myofibroblasts. Proc Natl Acad Sci U S A. 2010;107(46):20009-20014.

36. Hodi FS, O'Day SJ, McDermott DF, et al. Improved survival with ipilimumab in patients with metastatic melanoma. $N$ Engl J Med. 2010;363(13):1290.

37. Tatsumi T, Takehara T, Katayama K, et al. Expression of costimulatory molecules B7-1 (CD80) and B7-2 (CD86) on human hepatocellular carcinoma. Hepatology. 1997;25(5):1108-1114.

38. Shi F, Shi M, Zeng Z, et al. PD-1 and PD-L1 upregulation promotes CD8(+) T-cell apoptosis and postoperative recurrence in hepatocellular carcinoma patients. Int J Cancer. 2011;128(4):887-896.

39. Li FJ, Zhang Y, Jin GX, Yao L, Wu DQ. Expression of LAG-3 is coincident with the impaired effector function of HBV-specific CD8(+) T cell in HCC patients. Immunol Lett. 2013;150(1-2):116-122.

40. Li H, Wu K, Tao K, et al. Tim-3/galectin-9 signaling pathway mediates T-cell dysfunction and predicts poor prognosis in patients with hepatitis B virus-associated hepatocellular carcinoma. Hepatology. 2012;56(4):1342-1351.

41. Stemmer SM, Benjaminov O, Medalia G, et al. CF102 for the treatment of hepatocellular carcinoma: a phase I/II, open-label, dose-escalation study. Oncologist. 2013;18(1):25-26.

42. Pedroza-Gonzalez A, Kwekkeboom J, Sprengers D. T-cell suppression mediated by regulatory $T$ cells infiltrating hepatic tumors can be overcome by GITRL treatment. Oncoimmunology. 2013;2(1):e22450.

43. Pedroza-Gonzalez A, Zhou G, Singh SP, et al. GITR engagement in combination with CTLA-4 blockade completely abrogates immunosuppression mediated by human liver tumor-derived regulatory $\mathrm{T}$ cells ex vivo. Oncoimmunology. 2015;4(12):e1051297.

44. Melero I, Hervas-Stubbs S, Glennie M, Pardoll DM, Chen L. Immunostimulatory monoclonal antibodies for cancer therapy. Nat Rev Cancer. 2007;7(2):95-106.

45. Hoos A, Ibrahim R, Korman A, et al. Development of ipilimumab: contribution to a new paradigm for cancer immunotherapy. Semin Oncol. 2010;37(5):533-546.

46. Weber J. Immune checkpoint proteins: a new therapeutic paradigm for cancer - preclinical background: CTLA-4 and PD-1 blockade. Semin Oncol. 2010;37(5):430-439.

47. Linsley PS, Brady W, Urnes M, Grosmaire LS, Damle NK, Ledbetter JA. CTLA-4 is a second receptor for the B cell activation antigen B7. J Exp Med. 1991;174(3):561-569.
48. Engelhardt JJ, Sullivan TJ, Allison JP. CTLA-4 overexpression inhibits T cell responses through a CD28-B7-dependent mechanism. J Immunol. 2006;177(2):1052-1061.

49. Waterhouse P, Penninger JM, Timms E, et al. Lymphoproliferative disorders with early lethality in mice deficient in Ctla-4. Science. 1995;270(5238):985-988.

50. Flecken T, Schmidt N, Hild S, et al. Immunodominance and functional alterations of tumor-associated antigen-specific CD8+T-cell responses in hepatocellular carcinoma. Hepatology. 2014;59(4):1415-1426.

51. Gehring AJ, Ho ZZ, Tan AT, et al. Profile of tumor antigen-specific CD8 T cells in patients with hepatitis B virus-related hepatocellular carcinoma. Gastroenterology. 2009;137(2):682-690.

52. Chen Z, Shen S, Peng B, Tao J. Intratumoural GM-CSF microspheres and CTLA-4 blockade enhance the antitumour immunity induced by thermal ablation in a subcutaneous murine hepatoma model. Int $J$ Hyperthermia. 2009;25(5):374-382.

53. Sangro B, Gomez-Martin C, de la Mata M, et al. A clinical trial of CTLA-4 blockade with tremelimumab in patients with hepatocellular carcinoma and chronic hepatitis C. J Hepatol. 2013;59(1):81-88.

54. Sprinzl MF, Galle PR. Facing the dawn of immunotherapy for hepatocellular carcinoma. J Hepatol. 2013;59(1):9-10.

55. Jin HT, Anderson AC, Tan WG, et al. Cooperation of Tim-3 and PD-1 in CD8 T-cell exhaustion during chronic viral infection. Proc Natl Acad Sci U S A. 2010;107(33):14733-14738.

56. Blackburn SD, Shin H, Haining WN, et al. Coregulation of CD8+ T cell exhaustion by multiple inhibitory receptors during chronic viral infection. Nat Immunol. 2009;10(1):29-37.

57. Ishida Y, Agata Y, Shibahara K, Honjo T. Induced expression of PD-1, a novel member of the immunoglobulin gene superfamily, upon programmed cell death. EMBO J. 1992;11(11):3887-3895.

58. Freeman GJ, Long AJ, Iwai Y, et al. Engagement of the PD-1 immunoinhibitory receptor by a novel B7 family member leads to negative regulation of lymphocyte activation. J Exp Med. 2000;192(7):1027-1034.

59. Keir ME, Liang SC, Guleria I, et al. Tissue expression of PD-L1 mediates peripheral T cell tolerance. J Exp Med. 2006;203(4):883-895.

60. Tseng SY, Otsuji M, Gorski K, et al. B7-DC, a new dendritic cell molecule with potent costimulatory properties for T cells. J Exp Med. 2001;193(7):839-846.

61. Dong H, Strome SE, Salomao DR, et al. Tumor-associated B7-H1 promotes T-cell apoptosis: a potential mechanism of immune evasion. Nat Med. 2002;8(8):793-800.

62. Wang L, Pino-Lagos K, de Vries VC, Guleria I, Sayegh MH, Noelle RJ. Programmed death 1 ligand signaling regulates the generation of adaptive Foxp3+CD4+ regulatory T cells. Proc Natl Acad Sci U S A. 2008;105(27):9331-9336.

63. Pardoll DM. The blockade of immune checkpoints in cancer immunotherapy. Nat Rev Cancer. 2012;12(4):252-264.

64. Willimsky G, Schmidt K, Loddenkemper C, Gellermann J, Blankenstein T. Virus-induced hepatocellular carcinomas cause antigen-specific local tolerance. J Clin Invest. 2013;123(3):1032-1043.

65. Wherry EJ. T cell exhaustion. Nat Immunol. 2011;12(6):492-499.

66. Hofmeyer KA, Jeon H, Zang X. The PD-1/PD-L1 (B7-H1) pathway in chronic infection-induced cytotoxic T lymphocyte exhaustion. J Biomed Biotechnol. 2011;2011:451694.

67. Gao Q, Wang XY, Qiu SJ, et al. Overexpression of PD-L1 significantly associates with tumor aggressiveness and postoperative recurrence in human hepatocellular carcinoma. Clin Cancer Res. 2009;15(3): 971-979.

68. El-Khoueiry AB, Melero I, Crocenzi TS, et al. Phase I/II safety and antitumor activity of nivolumab in patients with advanced hepatocellular carcinoma (HCC): CA209-040. ASCO Meeting Abstracts. 2015;33(Suppl):LBA101. 
Cancer Management and Research

\section{Publish your work in this journal}

Cancer Management and Research is an international, peer-reviewed open access journal focusing on cancer research and the optimal use of preventative and integrated treatment interventions to achieve improved outcomes, enhanced survival and quality of life for the cancer patient

The manuscript management system is completely online and includes

Submit your manuscript here: https://www.dovepress.com/cancer-management-and-research-journal 